\title{
Meta-Analysis of Synovial Fluid Polymerase Chain Reaction for Diagnosing Periprosthetic Hip and Knee Infection
}

\section{Cheng Li}

Charité Universitätsmedizin Berlin https://orcid.org/0000-0002-4080-8886

Hao Li

General Hospital of People's Liberation Army

Xue Yang

General Hospital of People's Liberation Army

Fang-Zheng Zhu

General Hospital of People's Liberation Army

Chi Xu

General Hospital of People's Liberation Army

Andrej Trampuz ( $\nabla$ andrej.trampuz@charite.de )

Charité Universitätsmedizin Berlin

\section{Research Article}

Keywords: Meta-analysis, diagnosis, arthroplasty, periprosthetic joint infection, synovial fluid, polymerase chain reaction

Posted Date: June 29th, 2021

DOI: https://doi.org/10.21203/rs.3.rs-564463/v1

License: () (7) This work is licensed under a Creative Commons Attribution 4.0 International License. Read Full License

Version of Record: A version of this preprint was published at Journal of Orthopaedic Surgery and Research on January 4th, 2022. See the published version at https://doi.org/10.1186/s13018-021-02813-8. 


\section{Abstract}

BACKGROUND AND OBJECTIVE: The purpose of this study was to estimate the diagnostic performance of synovial fluid polymerase chain reaction (PCR) in periprosthetic hip and knee infection, and whether synovial fluid PCR has greater diagnostic significance than conventional methods.

METHODS: The literature databases PubMed, Scopus, and the Web of Science were searched for English articles describing periprosthetic joint infection (PJI) diagnosis by synovial fluid PCR. Articles were limited to the period between January 1990 and December 2019. Subsequently, conventional methods that were used on at least two occasions were included for further analysis. Data analysis was performed using the Meta-DiSc and Stata software.

RESULTS: Eleven studies with 1360 cases were included in the meta-analysis. The pooled sensitivity, specificity, and diagnostic odds ratio (DOR) of synovial fluid PCR were 0.70 (95\% Cl: 0.66-0.74), 0.92 (95\% Cl: 0.90-0.93), and 37.4 (95\% Cl: 17.77-78.74), respectively.

CONCLUSIONS: Synovial fluid PCR can be used as a diagnostic confirmatory method for PJI in cases of negative synovial fluid culture with highly suspected infection during the early stages.

\section{Background}

Periprosthetic joint infection (PJI) is one of the most severe complication following hip or knee arthroplasty, with high morbidity, mortality and costs [1-5]. However, its diagnosis remains a challenge in the management of PJI. To date, there is no test available that rapidly and accurately diagnoses infection with a sensitivity and specificity of $100 \%$ [6]. A mixture of several diagnostic methods or diagnostic definition that guide the diagnosis of PJI is currently the most common approach used [7].

Joint aspiration is an invasive diagnostic method and often used as the first step in suspected PJI cases. Currently, various synovial fluid tests have been applied in the clinical diagnosis of PJI [8,9]. Non-microbiological analysis of the synovial fluid white cell count and polymorphonuclear (PMN) leukocytes, alpha-defensin, leukocyte esterase (LE), and C-reactive protein (CRP) were included in the new definition of PJI from the Musculoskeletal Infection Society (MSIS) guideline of 2018 [10]. Although these tests are of high diagnostic value for PJI cases, they are unable to identify the causative pathogen. Conventional synovial fluid culture still appears to be an irreplaceable diagnostic approach for the detection of microorganisms. In recent years, polymerase chain reaction (PCR) was also used in the diagnosis of PJI. A number of reports found that synovial fluid PCR could rapidly detect rare bacterial infections, which is in contrast to conventional synovial fluid culture [11,12]. However, it remains unknown whether better diagnostic results are observed using PCR compared to tissue culture, with several conclusions arguable $[13,14]$. Although a meta-analysis of PCR for diagnosis of PJI has been previously performed using results combined from synovial fluid, periprosthetic tissue, and sonicate fluid samples [15], the diagnostic value of synovial fluid PCR in the periprosthetic hip and knee remains unclear.

The aim of the present meta-analysis was to assess the diagnostic accuracy of synovial fluid PCR in periprosthetic hip and knee infection. Furthermore, we examined whether synovial fluid PCR has a better diagnostic value compared to conventional methods, especially synovial fluid culture.

\section{Methods}

Search strategy

A systematic literature search of the electronic databases of Web of Science, PubMed, and Scopus was performed for manuscripts of English language between January 1990 and December 2019. The following medical subject headings (MeSH) or text keywords were used: "arthroplasty or joint prosthesis or joint replacement or periprosthetic joint or prosthetic joint", "infection or infectious or infected", "synovial fluid", and "PCR or polymerase chain reaction".

Two authors (LC and LH) independently selected research papers according to the following inclusion criteria: (1) human studies related to synovial fluid PCR in periprosthetic hip or knee infection; (2) clear description of the diagnosis standard of PJl; and (3) values on true-positive (TP), false-positive (FP), truenegative (TN), and false-negative (FN) were provided or could be computed.

The combined database was imported to EndNote X7 (Thomson Reuters, New York, NY, USA, 2013). All relevant publications on the synovial fluid PCR method in detecting periprosthetic hip or knee joint infection and their reference list were reviewed. Furthermore, included studies from two previous meta-analyses of synovial PCR were reviewed $[15,16]$. In addition, other diagnostic methods that appeared accumulatively on at least two occasions in all studies were collected. Diagnostic classification values of TP, FP, TN, and FN were included and further compared with synovial fluid PCR.

\section{Data extraction and study quality assessment}

Characteristics of the included studies were collected by two reviewers independently and assessed subsequently by a third reviewer. The following information was extracted: first author, year of publication, country, study design, number of total cases, infection site, acquisition time, diagnostic criteria, type of PCR, target gene, antimicrobial use before specimen collection, and diagnostic sensitivity and specificity. The quality of all identified synovial fluid studies was evaluated using the Quality Assessment of Diagnostic Accuracy Studies (QUADAS-2) guidelines.

\section{Statistical analysis}

To estimate the diagnostic value of synovial fluid PCR for PJI detection, all statistical analyses were performed using Meta-DiSc (version 1.4, Unit of Clinical Biostatistics team, Madrid, Spain) and Stata software (version 14.0, StataCorp, College Station, TX, USA). $\mathrm{I}^{2}$ was calculated to evaluate heterogeneity among the studies. If $\mathrm{I}^{2}>50 \%$, the random-effects model was used. Meta-regression analyses were performed to further assess the potential source of heterogeneity, 
such as type of prosthesis, number of patients, acquisition time, antibiotic use, sample condition, diagnostic standard, and target gene. Deeks' funnel plot asymmetry test was used to evaluate potential publication bias.

\section{Results}

Search results

Of the identified 145 primary articles, two records were extracted from the reference list of synovial fluid PCR-related studies [17,18]. Fifty-one studies were excluded due to duplication reasons. A further 83 were excluded after further reviewing the title, abstract, and full text. Finally, a total of 11 studies were considered suitable for meta-analysis (Fig. 1) [13,14,17-25]. Characteristics of the studies included are summarized in Table 1. Among these studies, five diagnostic methods could be compared with synovial fluid PCR. The QUADAS-2 quality assessments for the included studies are shown in Fig. 2.

Diagnostic accuracy

The random-effects model was used to examine study heterogeneity, which was found for the sensitivity $\left(I^{2}=73.9 \%\right)$, specificity $\left(I^{2}=86.0 \%\right)$, positive likelihood ratio (PLR) $\left(I^{2}=77.4 \%\right)$, negative likelihood ratio (NLR) $\left(I^{2}=62.2 \%\right)$, and diagnostic odds ratio (DOR) $\left(I^{2}=66.4 \%\right)$. The pooled sensitivity, specificity, PLR, NLR, and DOR estimates for the diagnosis of PJI using synovial fluid PCR were 0.70 (95\% Cl: 0.66-0.74), 0.92 (95\% Cl: 0.90-0.93), 9.09 (95\% Cl: 5.2815.67 ), 0.32 (95\% Cl: 0.24-0.42), and 37.4 (95\% Cl: 17.77-78.74), respectively (Fig. 3-7). The summary receiver operating characteristic (SROC) plot showed the summary sensitivity and specificity and the $95 \%$ confidence and prediction regions, with an area under the curve (AUC) of 0.9252 (standard error of 0.0195 ; Fig. 8).

Synovial fluid PCR was shown to have a better sensitivity and specificity than preoperative examination of CRP and erythrocyte sedimentation rate (ESR). Similar sensitivities were observed for conventional synovial fluid culture (70\%) and PCR (69\%). However, the specificity of synovial fluid PCR was lower than that of synovial fluid culture ( $91 \%$ vs. $98 \%$, respectively). Intraoperative histology, periprosthetic tissue culture, and sonicate fluid demonstrated better sensitivity and specificity than synovial fluid PCR. Details of the diagnostic values of synovial fluid PCR and other preoperative and intraoperative examination are shown in Table 2.

\section{Meta-regression analysis}

Meta-regression analysis was performed in the group of diagnostic standards, acquisition time, number of patients, antibiotic use, and target gene (Table 3 ). Results showed that the most likely sources of heterogeneity were acquisition time and antibiotic treatment $(P<0.001)$. Compared with the diagnostic standards of MSIS, International Consensus Meeting (ICM), or European Bone and Joint Infection Society (EBJIS), other diagnostic criteria had a higher sensitivity of $0.85(95 \% \mathrm{Cl}, 0.72-0.98 ; P<0.05)$. A small sample size $(<100)$ was observed to have a higher sensitivity of $0.81(95 \% \mathrm{Cl}, 0.71-0.90)$ compared to studies including more than 100 patients with a sensitivity of $0.71(95 \% \mathrm{Cl}, 0.59-0.83)(P=0.01)$.

\section{Assessment of publication bias}

Deeks' funnel plot analysis did not identify a potential publication bias for synovial fluid PCR $(P=0.41 ;$ Fig. 9$)$.

\section{Discussion}

The present meta-analysis showed that the pooled sensitivity and specificity of synovial fluid PCR are $70 \%$ and $92 \%$, respectively. The AUC value of the SROC was 0.9252 . These results suggest that synovial fluid PCR could be used for the diagnosis of infection after hip and knee arthroplasty. The first meta-analysis of the use of PCR in the diagnosis of PJI presented the sensitivity and specificity of synovial fluid ( $84 \%$ and $89 \%$, respectively), tissue samples ( $95 \%$ and $81 \%$, respectively), and sonicate fluid ( $81 \%$ and $96 \%$, respectively) [16], with moderate sensitivity and specificity levels observed for the three sample types. Interestingly, similar results were also reported by the retrospective study performed by Huang and colleagues [17]. In 2018, Jun et al. [15] performed a diagnostic meta-analysis of PCR in PJI, reporting a sensitivity of 0.76 (95\% Cl: $0.65-0.85)$ and specificity of 0.94 (95\% Cl: $0.92-0.95)$. Unfortunately, the pooled result combines samples from synovial fluid, sonicate fluid, and intraoperative tissue. Hence, the diagnostic value of synovial fluid PCR in periprosthetic hip and knee by meta-analysis remained unexplored. The present study is the first meta-analysis evaluating the diagnostic value of synovial fluid PCR for diagnosing infection after hip and knee replacement.

In recent years, the use of joint fluid for diagnosing PJI was a topic of considerable interest. Multiple synovial fluid tests were applied in clinical practice, with several valuable tests incorporated into the new definition of MSIS [10]. Through a literature review of the published meta-analysis of these synovial fluid methods from the MSIS guideline [26-30], the pooled sensitivity of these tests is superior to that of synovial fluid PCR. The specificity of synovial fluid PCR exceeded that of synovial fluid interleukin-6 (IL-6), CRP, WBC, and PMN, but was lower than synovial fluid culture, alpha-defensins, and LE. Based on the AUC value of meta-analysis of synovial fluid [31], only LE demonstrated excellent accuracy in the diagnosis of PJI, followed by alpha-defensins, IL-6, CRP, and PMN. Furthermore, synovial fluid WBC as well as PCR showed good accuracy (Table 4). Although the results of most synovial fluid biomarkers were superior to that of the synovial fluid PCR [26-30], synovial fluid culture and some PCR tests can detect bacteria and could provide a more valuable reference for further comparisons with intraoperative diagnostic results. However, the diagnostic value between synovial fluid culture and PCR for PJI detection is controversial.

Synovial fluid PCR was compared with preoperative and intraoperative tests from the included studies The PCR test was found to have better sensitivity and specificity than that of serum CRP and ESR. In contrast, lower sensitivity and specificity were observed in comparison to all intraoperative methods. Compared with synovial fluid culture, synovial fluid PCR had an almost identical level of sensitivity with synovial fluid culture (69\% vs. $70 \%$, respectively) and a lower specificity level ( $91 \%$ vs. 98\%, respectively). Synovial fluid PCR and culture were also observed to have similar results in comparison to the previous meta- 
analysis of synovial fluid culture, with a sensitivity of $70 \%$ and $72 \%$, respectively, and a specificity of $92 \%$ and $95 \%$, respectively [30]. Although the current meta-analysis and our subgroup results showed that the sensitivity and specificity of synovial fluid PCR were lower than that of synovial fluid, PCR has several advantages in regard to the detection of bacteria. Synovial fluid PCR has been reported to rapidly provide results within 3-72 $\mathrm{h}$ [14,23,25], and could also detect culture-negative bacteria $[11,12,21,22,25]$. Sujeesh and co-workers reported that the sensitivity of $16 \mathrm{~S}$ rRNA PCR and synovial fluid culture was $68.1 \%$ and $70.2 \%$, respectively [22]. PJI was detected by PCR in five cases that were negative by synovial fluid culture. Synovial fluid multiplex PCR identified 12 cases negative by synovial fluid culture, with 10 cases caused by low-virulence bacteria (coagulase-negative staphylococci and Cutibacterium acnes) [25]. The author also found that more cases of polymicrobial infections were detected by synovial PCR than synovial fluid culture (four vs. two cases), with similar results also reported by Melendez and colleagues [18,21]. Due to rare cases of mixed infection in these studies, further research is required. However, in cases treated with antibiotics before specimen collection, the testability of synovial fluid PCR was lower than synovial fluid culture. A comparison of the PCR panel and synovial fluid culture in patients that received antibiotics within 30 days before joint aspiration revealed the sensitivity of the PCR panel and synovial culture to be $64.5 \%$ and $85.4 \%$, respectively [21]. In another study, PCR-ESI/MS detected eight of nine PJI cases who had received antibiotics within 30 days, whereas synovial fluid culture detected all nine cases [18]. The use of antibiotics before PCR analysis most likely impacts culture results. Meta-regression analysis results from the current study show that the sensitivity level of cases receiving antibiotic therapy were less than cases without antibiotic therapy (73\% vs. 80\%). Moreover, meta-regression was also analyzed in the preoperative and intraoperative aspiration groups, with slightly higher sensitivity and lower specificity observed for the intraoperative test compared to the preoperative test (sensitivity: $77 \%$ vs. $75 \%$, respectively; specificity: $93 \%$ vs. $96 \%$ ). In contrast, the meta-analysis of synovial fluid white cell count performed by Qu and co-workers [32] found that preoperative collection had a higher sensitivity than intraoperative samples ( $91 \%$ vs. $77 \%$, respectively), and lower specificity than that of intraoperative samples (89\% vs. $97 \%$ ). However, due to the limited data of the studies included from our intraoperative study (two studies) and different tests performed in these two meta-analyses, whether intraoperative and preoperative sample collection infers with the diagnostic accuracy remains an avenue for further exploration.

Although various types of synovial fluid PCR have been tested in the clinical diagnosis of PJI, the diagnostic ability has most likely been disregarded. The most frequently described disadvantage of PCR is FP results, with the potential impacting factors, including the use of different target genes, PCR type, laboratory technician skills, and laboratory conditions $[13,19,22,24]$. The use of $16 S / 28 S$ rRNA RT-PCR with high-quality control standards demonstrated excellent results, with a sensitivity of $100 \%$ and specificity of $99.5 \%$ [13].Sebastian and colleagues [22] found that DNase treatment could reduce exogenous bacterial contamination, with no FP results observed in synovial fluid PCR: however, the sensitivity was affected. Further studies are required to determine the most suitable type of PCR for PJI diagnosis and the standard procedure required.

The present study has several limitations. First, the identified studies used different types of PCR; therefore, the overall result may impact the estimates of diagnostic accuracy. Second, meta-regression analysis was not performed in regard to the prosthesis type or sample condition to further explore sources of heterogeneity. The type of prosthesis described in the included studies were the knee or both the hip and knee; however, studies focusing only on the periprosthetic hip were not found. Therefore, further analysis of differences between the hip and knee could not be performed in meta-regression analysis. Regarding the sample condition, frozen specimens were used in the studies; however, there was ambiguity in terms of the use of fresh samples. Third, the diagnostic accuracy of synovial fluid PCR may be affected by the standard definition of PJI [33].

\section{Conclusions}

The diagnostic capability of synovial fluid PCR is not superior to that of synovial fluid culture. However, in cases of negative synovial fluid culture with highly suspected early-stage infection, synovial fluid PCR can be used as a rapid, diagnostic confirmatory tool.

\section{Abbreviations}

AUC: Area under the curve

C: Clinical signs of infection

Cl: Confidence interval

CRP: C-reactive protein

DOR: Diagnostic odds ratio

EBJIS: European Bone and Joint Infection Society

ESI/MS: Electrospray ionization mass spectrometry

ESR: Erythrocyte sedimentation rate

FN: False-negative

FP: False-positive

H: Histological examination

IDSA: Infectious Diseases Society of America 
ICM: International Consensus Meeting

IL-6: Interleukin-6

LE: Leukocyte esterase

M: Microbiological or laboratory examination

MeSH: Medical subject headings

MSIS: Musculoskeletal Infection Society

NLR: Negative likelihood ratio

N: No

NA: Not available

P: Presence sinus tract or purulence around the prosthesis

PCR: Polymerase chain reaction

PJI: Periprosthetic joint infection

PLR: Positive likelihood ratio

PMN: Polymorphonuclear

QUADAS-2: Quality Assessment of Diagnostic Accuracy Studies - 2

qPCR: Quantitative PCR

R: Radiographic images

RT: Real-time

SROC: Summary receiver operating characteristic

SE: Standard error

Sen: Sensitivity

Spe: Specificity

TN: True negative

TP: True positive

WBC: White blood cell count

Y: Yes

\section{Declarations}

\section{Funding}

PRO-IMPLANT Foundation

\section{Acknowledgements}

This work was supported by the PRO-IMPLANT Foundation, Berlin, Germany (https://www.pro-implant-foundation.org), a non-profit organization supporting research, education, global networking and care of patients with bone, joint or implant-associated infection.

\section{Competing interests}

The authors declare that they have no competing interests.

\section{References}

1. DelanoisRE,MistryJB,GwamCU,MohamedNS,ChoksiUS,MontMA.Current Epidemiology of Revision Total Knee Arthroplasty in the United States.J Arthroplasty.2017;32:2663-8. 
2. GwamCU,MistryJB,MohamedNS,ThomasM,BigartKC,MontMA,etal.Current Epidemiology of Revision Total Hip Arthroplasty in the United States: National Inpatient Sample 2009 to 2013.J Arthroplasty.2017;32:2088-92.

3. KurtzSM,LauE,WatsonH,SchmierJK,ParviziJ.Economic burden of periprosthetic joint infection in the United States.J Arthroplasty.2012;27:61-5.e1.

4. NatsuharaKM,SheltonTJ,MeehanJP,LumZC.Mortality During Total Hip Periprosthetic Joint Infection.J Arthroplasty.2019;34:S337-42.

5. LumZC,NatsuharaKM,SheltonTJ,GiordaniM,PereiraGC,MeehanJP.Mortality During Total Knee Periprosthetic Joint Infection.J Arthroplasty.2018;33:37838.

6. LiC,Ojeda-ThiesC,XuC,TrampuzA.Meta-analysis in periprosthetic joint infection: a global bibliometric analysis.J Orthop Surg Res.2020;15:251.

7. LiC,RenzN,TrampuzA.Management of Periprosthetic Joint Infection.Hip Pelvis.2018;30:138-46.

8. GollwitzerH,DombrowskiY,ProdingerPM,PericM,SummerB,HapfelmeierA,etal.Antimicrobial peptides and proinflammatory cytokines in periprosthetic joint infection.J Bone Joint Surg Am.2013;95:644-51.

9. KarbyshevaS,YermakK,GrigorichevaL,RenzN,PerkaC,TrampuzA.SynovialFluidd-Lactate-ANovelPathogenSpecificBiomarkerfortheDiagnosisofPeriprostheticJointInfection.JArthroplasty[Internet].2020;Availablefrom:https://linkinghub.elsevier.com/retrieve/pii/S0

10. ParviziJ,TanTL,GoswamiK,HigueraC,Della ValleC,ChenAF,etal.The 2018 Definition of Periprosthetic Hip and Knee Infection: An Evidence-Based and Validated Criteria.J Arthroplasty.2018;33:1309-14.e2.

11. ChenouardR,HoppéE,LemariéC,TalhaA,DucellierF,FerchaudF,etal.A rare case of Prosthetic Joint Infection associated with Coxiella burnetii.Int $J$ Infect Dis.2019;87:166-9.

12. FarrellJJ,LarsonJA,AkesonJW,LoweryKS,RoundsMA,SampathR,etal.Ureaplasma parvum prosthetic joint infection detected by PCR.J Clin Microbiol.2014;52:2248-50.

13. KuoF-C,LuY-D,WuC-T,YouH-L,LeeG-B,LeeMS.Comparison of molecular diagnosis with serum markers and synovial fluid analysis in patients with prosthetic joint infection.Bone Joint J.2018;100-B:1345-51.

14. FinkB,SteurerM,HofäckerS,SchäferP,SandowD,SchusterP,etal.Preoperative PCR analysis of synovial fluid has limited value for the diagnosis of periprosthetic joint infections of total knee arthroplasties.Arch Orthop Trauma Surg.2018;138:871-8.

15. JunY,JianghuaL.Diagnosis of Periprosthetic Joint Infection Using Polymerase Chain Reaction: An Updated Systematic Review and Meta-Analysis.Surg Infect.2018;19:555-65.

16. QuX,ZhaiZ,LiH,LiH,LiuX,ZhuZ,etal.PCR-based diagnosis of prosthetic joint infection.J Clin Microbiol.2013;51:2742-6.

17. HuangZ,WuQ,FangX,LiW,ZhangC,ZengH,etal.Comparison of culture and broad-range polymerase chain reaction methods for diagnosing periprosthetic joint infection: analysis of joint fluid, periprosthetic tissue, and sonicated fluid.Int Orthop.2018;42:2035-40.

18. MelendezDP,UhlJR,Greenwood-QuaintanceKE,HanssenAD,SampathR,PatelR.DetectionofProstheticJointlnfectionbyUseofPCRElectrospraylonizationMassSpectrometryAppliedtoSynovialFluid[Internet].JournalofClinicalMicrobiology.2014.p.2202-

5.Availablefrom:http://dx.doi.org/10.1128/jcm.00570-14

19. BerginPF,DoppeltJD,HamiltonWG,MirickGE,JonesAE,SritulanondhaS,etal.Detection of periprosthetic infections with use of ribosomal RNA-based polymerase chain reaction.J Bone Joint Surg Am.2010;92:654-63.

20. LarsenLH,KhalidV,XUY,ThomsenTR,SchønheyderHC,the PRIS Study Group.DifferentialContributionsofSpecimenTypes,Culturing,and16SrRNASequencinginDiagnosisofProstheticJointInfections.JClinMicrobiol[Internet].2018;: 17

21. MelendezDP,Greenwood-QuaintanceKE,BerbariEF,OsmonDR,MandrekarJN,HanssenAD,etal.Evaluation of a Genus- and Group-Specific Rapid PCR Assay Panel on Synovial Fluid for Diagnosis of Prosthetic Knee Infection.J Clin Microbiol.2016;54:120-6.

22. SebastianS,MalhotraR,SreenivasV,KapilA,ChaudhryR,DhawanB.Utility of 16 S rRNA PCR in the Synovial Fluid for the Diagnosis of Prosthetic Joint Infection.Ann Lab Med.2018;38:610-2.

23. JanzV,SchoonJ,MorgensternC,PreiningerB,ReinkeS,DudaG,etal.Rapid detection of periprosthetic joint infection using a combination of $16 \mathrm{~s} r \mathrm{DNA}$ polymerase chain reaction and lateral flow immunoassay: A Pilot Study.Bone Joint Res.2018;7:12-9.

24. PanousisK,GrigorisP,Butcherl,RanaB,ReillyJH,HamblenDL.Poor predictive value of broad-range PCR for the detection of arthroplasty infection in 92 cases.Acta Orthop.2005;76:341-6.

25. MorgensternC,CabricS,PerkaC,TrampuzA,RenzN.Synovial fluid multiplex PCR is superior to culture for detection of low-virulent pathogens causing periprosthetic joint infection.Diagn Microbiol Infect Dis.2018;90:115-9.

26. ChenY,KangX,TaoJ,ZhangY,YingC,LinW.Reliability of synovial fluid alpha-defensin and leukocyte esterase in diagnosing periprosthetic joint infection (PJI): a systematic review and meta-analysis.J Orthop Surg Res.2019;14:453.

27. XieK,DaiK,QuX,YanM.Serum and Synovial Fluid Interleukin-6 for the Diagnosis of Periprosthetic Joint Infection.Sci Rep.2017;7:1496.

28. WangC,WangQ,LiR,DuanJ-Y,WangC-B.Synovial Fluid C-reactive Protein as a Diagnostic Marker for Periprosthetic Joint Infection: A Systematic Review and Meta-analysis.Chin Med J.2016;129:1987-93.

29. DeFineM,GiavaresiG,FiniM,IlluminatiA,TerrandoS,PignattiG. The role of synovial fluid analysis in the detection of periprosthetic hip and knee infections: a systematic review and meta-analysis.Int Orthop.2018;42:983-94.

30. QuX,ZhaiZ,WuC,JinF,LiH,WangL,etal.Preoperative aspiration culture for preoperative diagnosis of infection in total hip or knee arthroplasty.J Clin Microbiol.2013;51:3830-4. 
31. JonesCM,AthanasiouT.Summary receiver operating characteristic curve analysis techniques in the evaluation of diagnostic tests.Ann. Thorac. Surg.2005.p.16-20.

32. QuX,ZhaiZ,LiuX,LiH,WuC,LiY,etal.Evaluation of white cell count and differential in synovial fluid for diagnosing infections after total hip or knee arthroplasty.PLoS One.2014;9:e84751.

33. LiC,RenzN,TrampuzA,Ojeda-

ThiesC.Twentycommonerrorsinthediagnosisandtreatmentofperiprostheticjointinfection.IntOrthop[Internet].2019;Availablefrom:http://dx.doi.org/10.1007/s 019-04426-7

\section{Tables}

Due to technical limitations, table 1-4 is only available as a download in the Supplemental Files section.

\section{Figures}

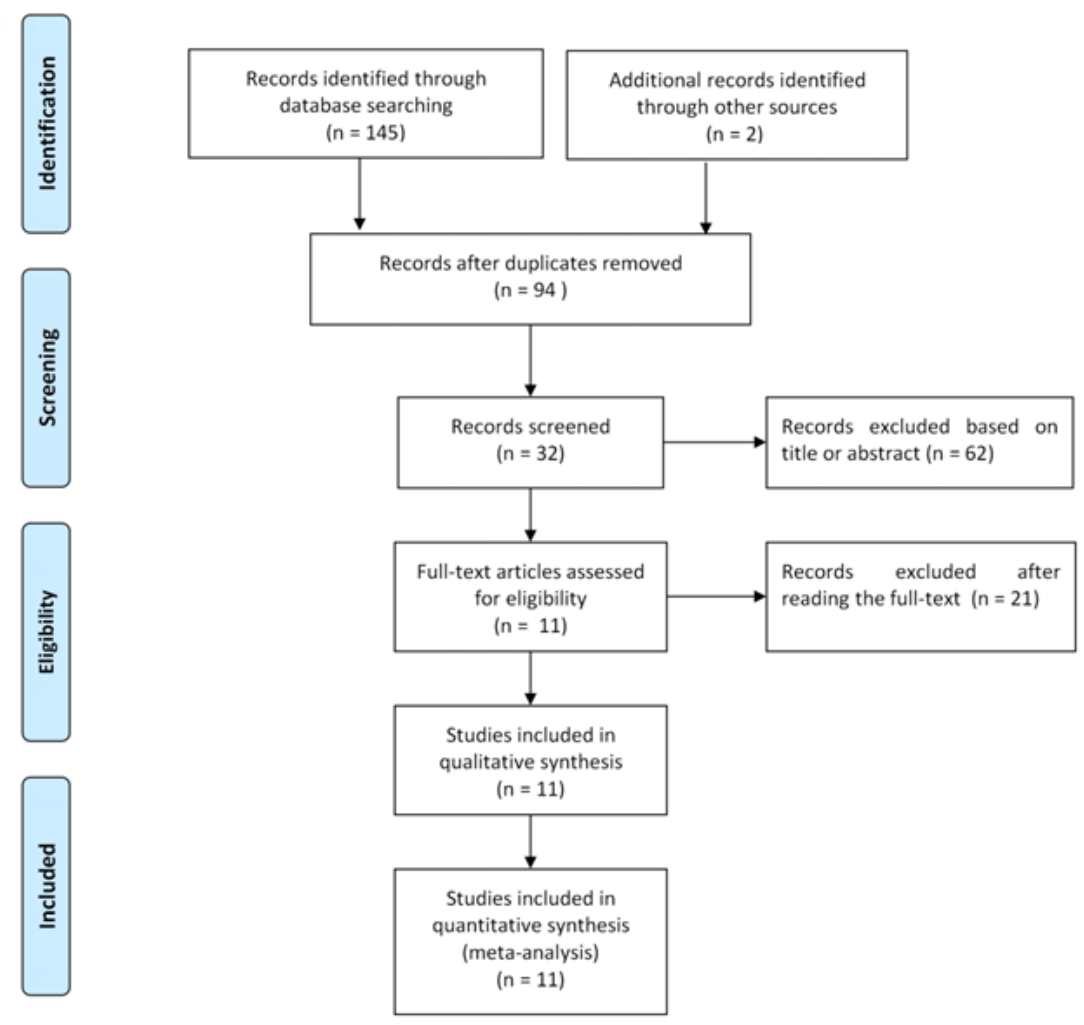

Figure 1

Flow diagram of the selection process for eligible studies. 


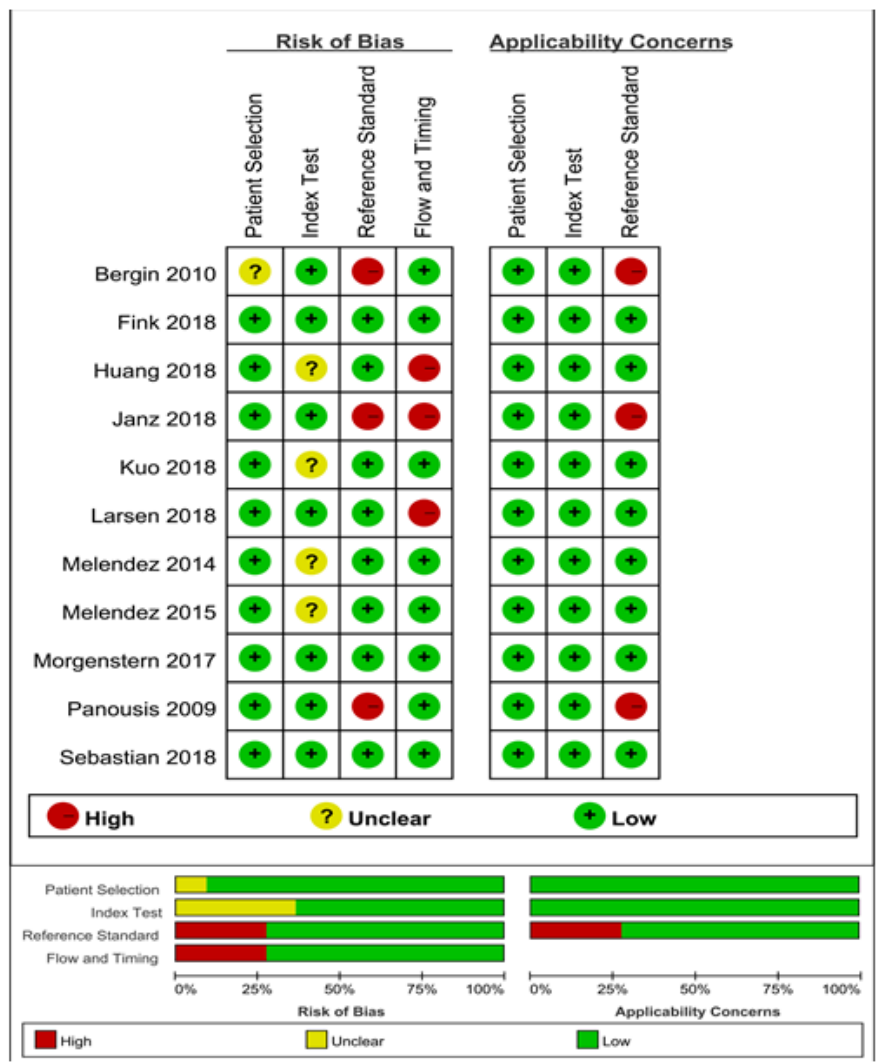

Figure 2

Methodological quality assessment of the included studies.

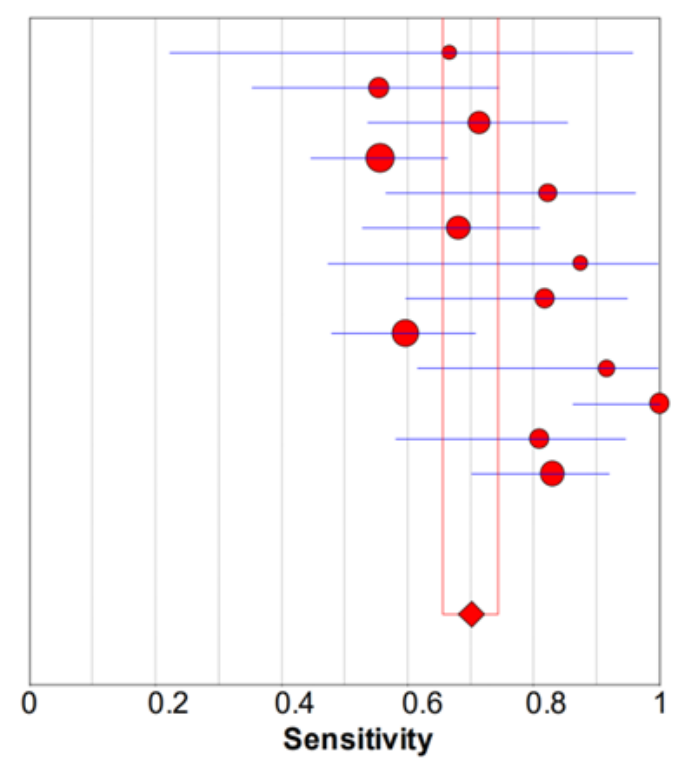

Sensitivity $(95 \% \mathrm{Cl})$

$0.67 \quad(0.22-0.96)$

$0.56 \quad(0.35-0.75)$

$0.71 \quad(0.54-0.85)$

$0.56 \quad(0.45-0.66)$

$0.82 \quad(0.57-0.96)$

$0.68 \quad(0.53-0.81)$

$0.88 \quad(0.47-1.00)$

$0.82 \quad(0.60-0.95)$

$0.60 \quad(0.48-0.71)$

$0.92 \quad(0.62-1.00)$

$1.00(0.86-1.00)$

$0.81(0.58-0.95)$

$0.83 \quad(0.70-0.92)$

Pooled Sensitivity $=0.70(0.66$ to 0.74$)$

Chi-square $=45.94 ; \mathrm{df}=12(\mathrm{p}=0.0000)$

Inconsistency (l-square) $=73.9 \%$

Figure 3

Forest plots of sensitivity of synovial fluid PCR for PJI diagnosis. 


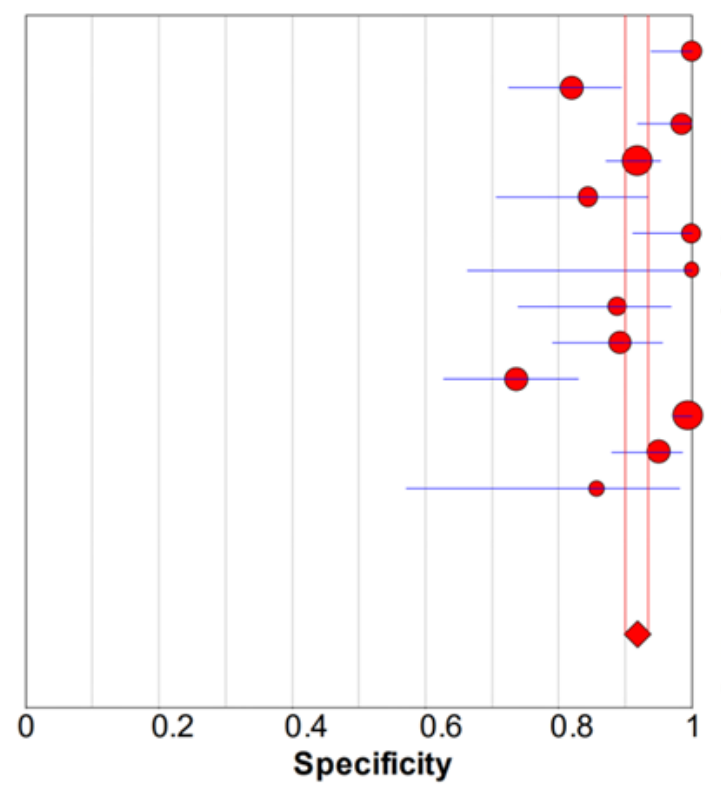

Specificity $(95 \% \mathrm{Cl})$

Bergin

Fink

Larsen

Melendez

Melendez

Sebastian

Janz

Morgenstern

Panousis

Kuo

Melendez

Huang

Pooled Specificity $=0.92(0.90$ to 0.93$)$

Chi-square $=85.94 ; \mathrm{df}=12(\mathrm{p}=0.0000)$

Inconsistency (-square) $=86.0 \%$

Figure 4

Forest plots of specificity of synovial fluid PCR for PJI diagnosis.

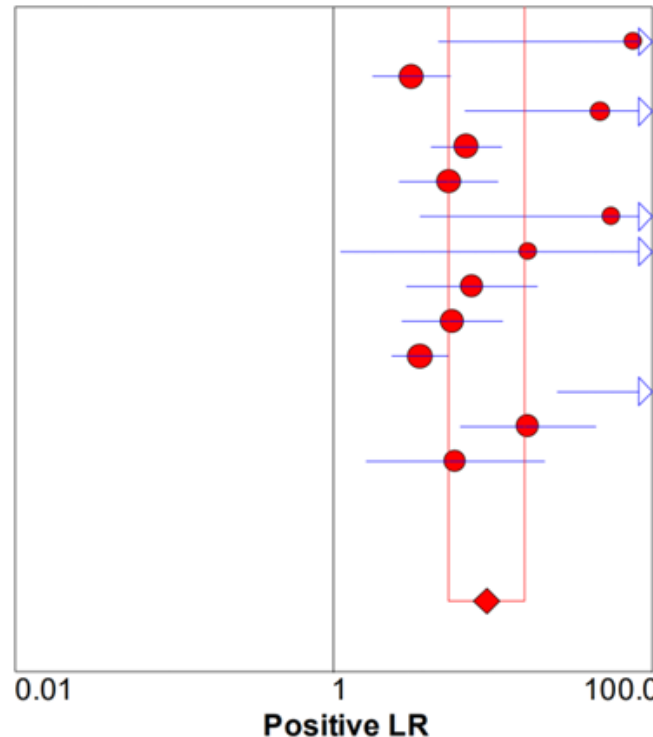

Bergin

Fink

Larsen

Melendez

Melendez

Sebastian

Janz

Janz

Morgenstern

Panousis

Kuo

Melendez

Huang
Positive LR (95\% CI)

$75.86 \quad(4.55-1,265.96)$

$3.09(1.77-5.40)$

$47.14 \quad(6.66-333.45)$

$6.82(4.12-11.30)$

$5.29 \quad(2.59-10.83)$

$55.52 \quad(3.51-878.78)$

$16.67 \quad(1.10-252.34)$

$7.36 \quad(2.86-18.94)$

$5.55(2.69-11.44)$

$3.49 \quad(2.33-5.24)$

$124.23 \quad(25.21-612.18)$

$16.60 \quad(6.24-44.13)$

$5.81 \quad(1.60-21.09)$

Random Effects Model

Pooled Positive LR = 9.09 (5.28 to 15.67)

Cochran- $Q=53.02 ; \mathrm{df}=12(\mathrm{p}=0.0000)$

Inconsistency (l-square) $=77.4 \%$

Tau-squared $=0.6135$

Figure 5

Forest plots of positive likelihood ratio of synovial fluid PCR for PJI diagnosis. 


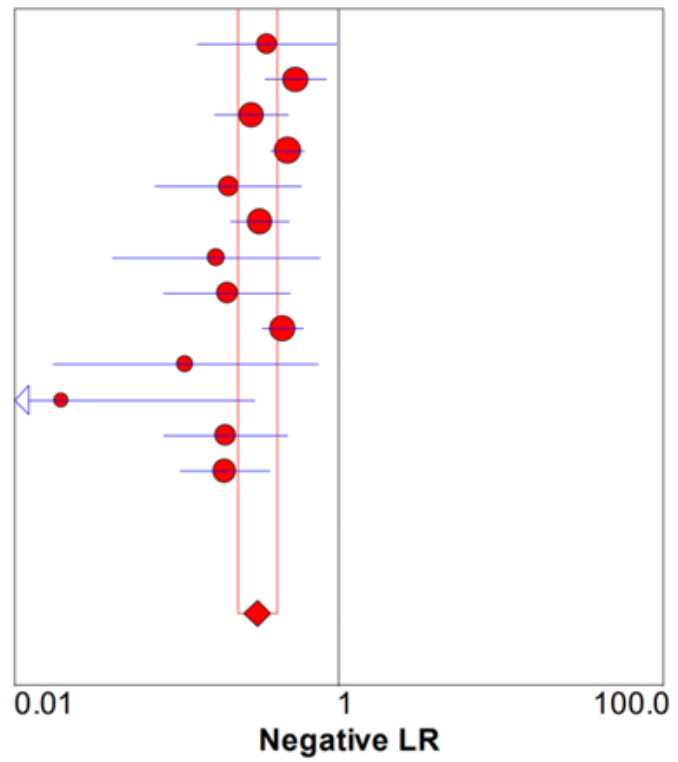

Negative LR (95\% CI)

$\begin{array}{llc}\text { Bergin } & 0.36 & (0.13-0.97) \\ \text { Fink } & 0.54 & (0.35-0.84) \\ \text { Larsen } & 0.29 & (0.17-0.49) \\ \text { Melendez } & 0.48 & (0.38-0.61) \\ \text { Melendez } & 0.21 & (0.07-0.59) \\ \text { Sebastian } & 0.33 & (0.22-0.49) \\ \text { Janz } & 0.18 & (0.04-0.76) \\ \text { Janz } & 0.20 & (0.08-0.50) \\ \text { Morgenstern } & 0.45 & (0.34-0.60) \\ \text { Panousis } & 0.11 & (0.02-0.74) \\ \text { Kuo } & 0.02 & (0.00-0.30) \\ \text { Melendez } & 0.20 & (0.08-0.48) \\ \text { Huang } & 0.20 & (0.11-0.37)\end{array}$

Random Effects Model

Pooled Negative LR $=0.32$ (0.24 to 0.42$)$

Cochran- $Q=31.73 ; \mathrm{df}=12(\mathrm{p}=0.0015)$

Inconsistency (t-square) $=62.2 \%$

Tau-squared $=0.1215$

Figure 6

Forest plots of negative likelihood ratio of synovial fluid PCR for PJI diagnosis.

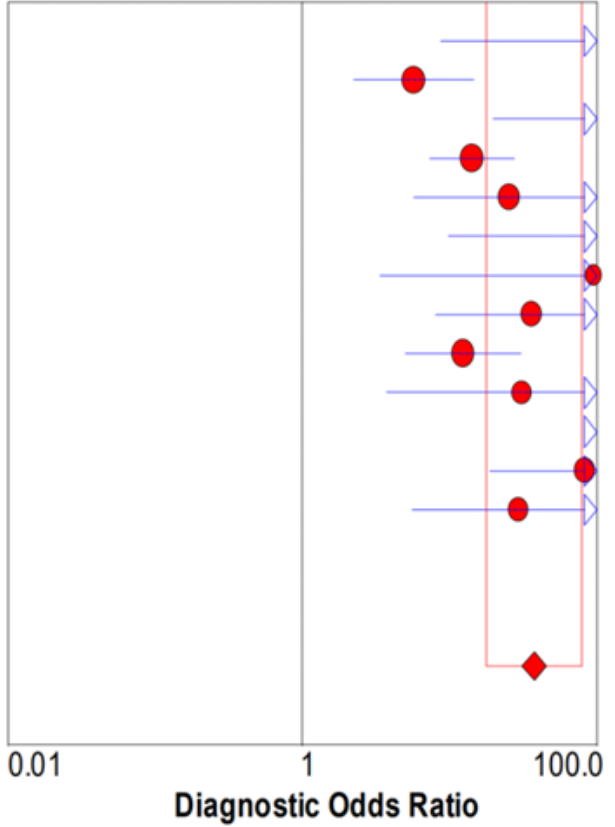

Bergin

Fink

Larsen

Melendez

Melendez

Sebastian

Janz

Janz

Morgenstern

Panousis

Kuo

Melendez

Huang

Random Effects Model

Pooled Diagnostic Odds Ratio $=37.40$ (17.77 to 78.74 )

Cochran- $Q=35.74 ; d f=12(p=0.0004)$

Inconsistency (l-square) $=66.4 \%$

Tau-squared $=1.0318$
Diagnostic OR $(95 \% \mathrm{Cl})$

$210.60 \quad(8.72-5,085.37)$

$5.70 \quad(2.25-14.49)$

$162.50 \quad(19.77-1,335.99)$

$14.13(7.29-27.40)$

$25.33 \quad(5.74-111.83)$

$169.84 \quad(9.79-2,947.15)$

$95.00 \quad(3.36-2,682.79)$

$36.00(8.02-161.57)$

$12.29(4.96-30.45)$

$30.90 \quad(3.76-254.10)$

$6,409.00 \quad(254.23-161,568.28)$

$82.88(18.83-364.75)$

$29.33(5.58-154.25)$

\section{Figure 7}

Forest plots of diagnostic odds ratio of synovial fluid PCR for PJI diagnosis. 


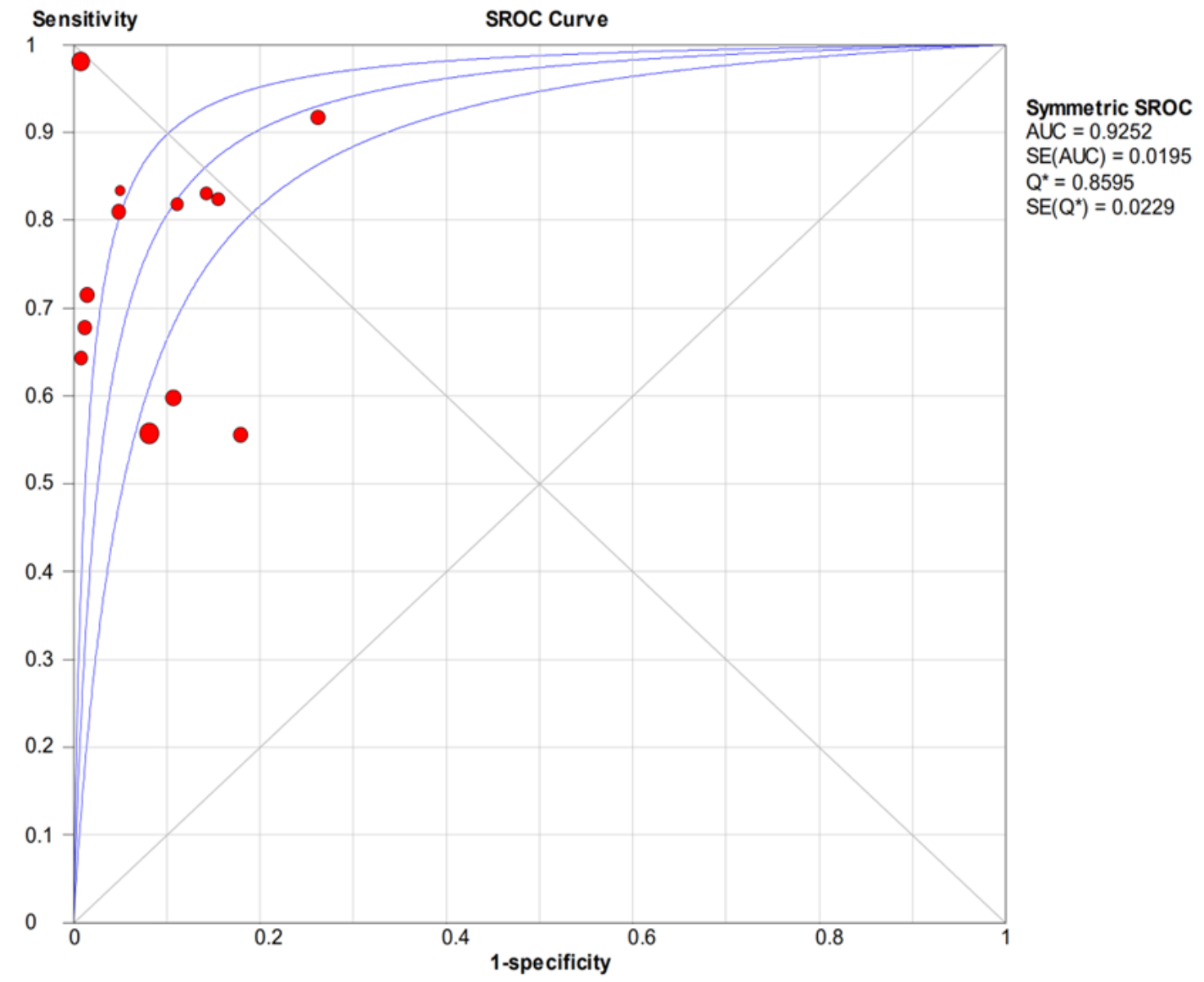

Figure 8

Summary of SROC of synovial fluid PCR for PJI diagnosis. 


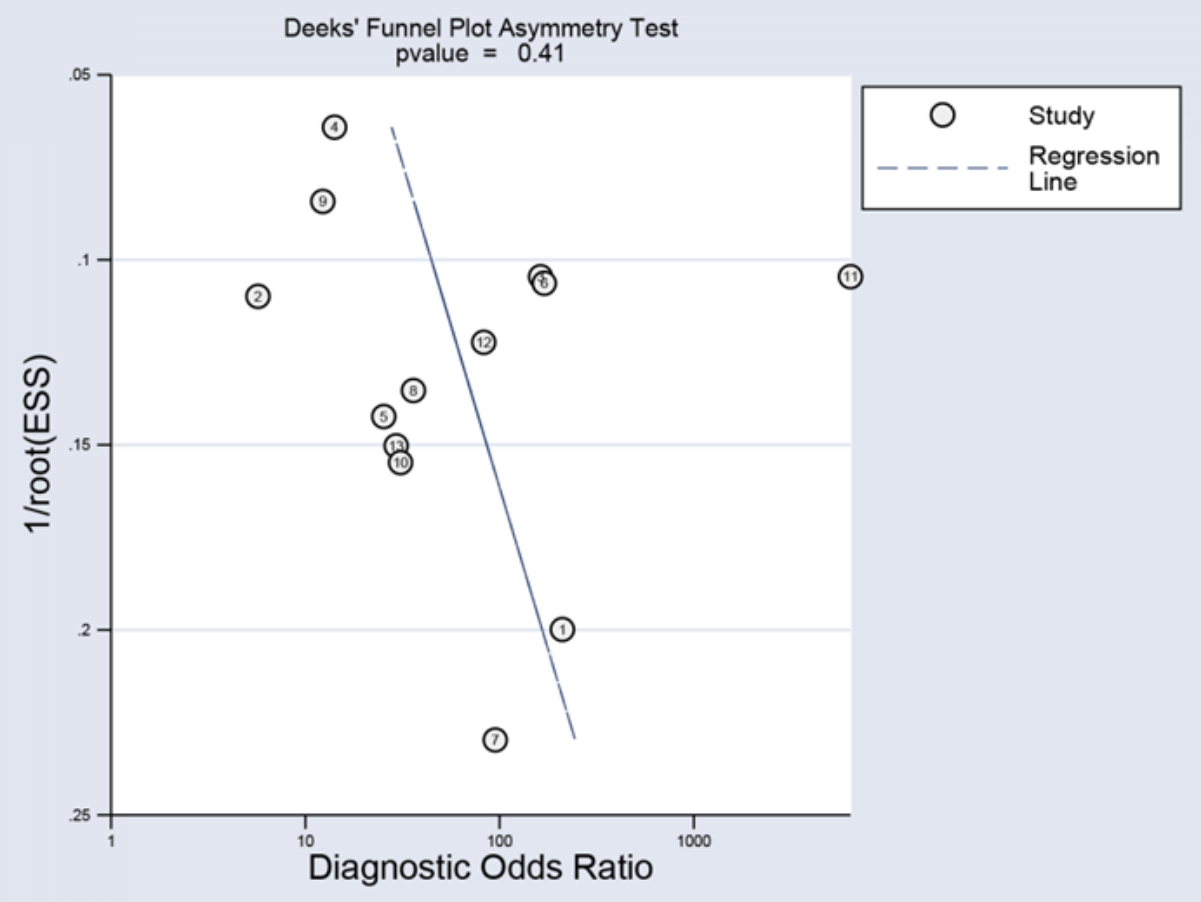

Figure 9

Deeks' funnel plot for evaluation of publication bias.

\section{Supplementary Files}

This is a list of supplementary files associated with this preprint. Click to download.

- Tables.pdf 